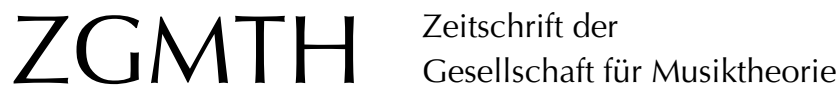

Schläbitz, Norbert (2011): Musiktheorie und veränderte Praxis: Anders sein, als man ist. ZGMTH 8/1, 39-42. https://doi.org/10.31751/616

\section{(C) 2011 Norbert Schläbitz}

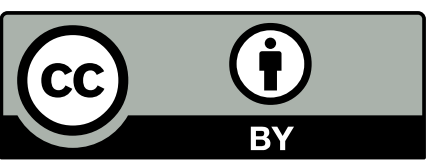

Dieser Text erscheint im Open Access und ist lizenziert unter einer Creative Commons Namensnennung 4.0 International Lizenz.

This is an open access article licensed under a

Creative Commons Attribution 4.0 International License.

veröffentlicht / first published: 14/06/2011

zuletzt geändert / last updated: 02/07/2013 


\title{
Musiktheorie und veränderte Praxis: Anders sein, als man ist.
}

\author{
Norbert Schläbitz
}

Der Name Musiktheorie trägt ein Programm in sich, eben jene zu verstehen als das theorie- (als auch praxis-)geleitete Nachdenken

1. über das Zusammenwirken von Klängen,

2. über die gesellschaftlichen und medialen Möglichkeitsbedingungen von Musik und

3. über den eigenen Reflexionsstandort, von dem aus Musik beleuchtet wird.

So verstanden geht Musiktheorie über bloße Harmonie-, Formenlehre oder Kontrapunkt weit hinaus, da hier zusammengedacht wird, was im Raum der Musikwissenschaft einst eine künstliche und nicht tragfähige oder gar begründbare Trennung erfahren hat.

Musiktheorie in diesem Sinne lehnt eine rein musikimmanente Betrachtung grundsätzlich ab. Die Vorstellung, Musik wäre immanent nach einer sie auszeichnenden Logik allein zu betrachten, und so der vielfach zitierte Satz von Carl Dahlhaus, »Der Triumph der Analyse [!] besteht in dem Nachweis, daß ein Werk, mindestens ein geglücktes, nicht anders sein kann, als es ist«, verfahren naiv und unterkomplex. Sie reduzieren Musik zu einem rein mathematisch berechenbaren Kalkulationsobjekt, das allein Algorithmen geschrieben gemäß vermeintlich ermittelter Gesetzmäßigkeiten - abarbeitet.

Wer demnach den Nachweis eines So-und-nicht-anders erbringen will, dürfte bei Fugen komponierenden Computerprogrammen eher fündig werden als beispielsweise bei Johann Sebastian Bach, der im Vergleich zu ersteren sich die Freiheit nahm, dem bloßen Schema Fuge seine individuelle Form auf-, und auf diese Weise dem realisierten Schema einen Mangel einzuprägen. Einen solchen Nachweis geführt zu haben, dürfte der Musik auch kaum zum Lob gereichen: Wo etwas »nicht anders sein kann, als es ist», stehen die Redundanz und musikalische Langeweile Pate. Die Differenz dagegen macht erkennbar eine "geglückte« Musik aus, denn in Musik ist nicht die Regel oder eine idealisierte musikalische Logik (im Sinne »allgemein gültige[r] Gesetze« [Hugo Riemann]) entscheidend, die sich erfüllt, sondern die Abweichung. In dem Ausloten eines vorgeblichen Soseins einer Musik ist so ein gravierender Systemfehler mitgeführt, der anerkanntermaßen aus dem Raum der Musik auch selbst der Kritik überstellt ist. Mit Blick auf jene zitierte Sentenz von Dahlhaus schreibt beispielsweise Martin Geck: »Die Betriebsblindheit, mit der dieser kluge, vielseitige und hochgebildete Gelehrte hier argumentiert, ist mit Händen zu greifen.«1

1 Geck 2009, 43. 
Wer immer komponiert, ist von ideengebenden und Gesellschaft konstituierenden Systemen im Sinne Luhmanns sowie - um eine weitere Theorie zu bemühen - von den 'Materialitäten der Kommunikation im Sinne Marshall McLuhans - hier in der Lesart Hans Ulrich Gumbrechts und Karl Ludwig Pfeiffers ${ }^{2}$ - umfangen, die sich paradox gesprochen - da Musik eine gänzlich nichtssagende Kunst ist - kommunikativ in Musik Ausdruck verleihen. Solche Musik generierende, durch reinen Bezug auf Notenereignisse, oftmals vernachlässigte Umwelten gilt es musiktheoretisch ebenfalls zu berücksichtigen. Das Analysieren und Verstehen von Musik bedarf daher eines erweiterten Blickwinkels.

Wenngleich begrüßenswert und doch unterkomplex agiert die Vorstellung, die den über die Musik hinausgehenden Kontext miteinbezieht, vernachlässigt sie doch dabei die Motive des Beobachters, der untersucht und Wertschätzungen ausspricht. Die Wertmaßstäbe des Beobachters bei jeder musiktheoretischen Betrachtung offenzulegen ist unverzichtbar, ändert sich doch mit jedem Wechsel der Perspektive der Maßstab, mit dem gemessen wird, und so auch das Urteil über Musik. Plakativ formuliert: Mit der Wertschätzung von Sinfonien bei Vertreten eines entsprechenden Maßstabes fällt das Urteil über Minimal Music oder auch über Populäre Musik entsprechend düster aus. Mit der Wertschätzung rhythmischer Gestalt fällt umgekehrt das Urteil über die abendländische Kunstmusik nicht gerade freundlich aus usw.

Es gibt keinen irgendwie gearteten Maßstab (sozusagen eine musikalische Weltformel ) für "geglückte« oder qualitativ hochstehende Musik, der sich theoretisch begründen ließe. Wer `Komplexitätı zum Maßstab für die Qualität von Musik erhebt, sieht sich vor das unlösbare Problem gestellt, zu erklären, wieso wachsende Komplexität nicht mit wachsender Qualität einhergehen muss und wieso manche klug durchkalkulierte hochkomplexe serielle Musik doch nicht so geglückt scheint wie manche Musik der Vergangenheit oder manche Musik der Gegenwart, die durch frappante Einfachheit ausgezeichnet ist.

2 Vgl. Gumbrecht/Pfeiffer 1988. Unter >Materialitäten der Kommunikation` werden alle Medien verstanden, die Kommunikation und deren Distribution ermöglichen: z. B.: (Noten-)Schrift, (Noten-) Papier, Buch, Film, Brief, Computer. Auch Medien wie Schreibmaschine, Füller, Bleistift u.v.a.m. gehören dazu. Musikinstrumente mit ihren Qualitäten lassen sich ebenfalls darunter subsumieren. Luhmann bezieht solche Materialitäten nicht in seine systemtheoretischen Überlegungen ein, da bei Luhmann aus dem Dreischritt ıInformation-Mitteilung-Verstehen Kontingenzı Kommunikation allein sich entfaltet. Ganz anders sieht dies bei McLuhan u. a. aus, die jenen Materialitäten essentielle Bedeutung bei der Generierung von Kommunikation zumessen dergestalt, dass mit dem Gebrauch spezifischer Materialitäten als Emergenzphänomen auch eine ganz spezifische folgenreiche Kommunikation einhergeht. Das aber wird in der Regel nicht offenkundig, denn wer Texte oder Noten liest, lenkt seine Aufmerksamkeit auf den Inhalt, sucht Sinn zu erschließen oder Strukturen zu analysieren und ist dabei ıblind ‘ür den Träger der Information. Dass der Träger einer Information aber Inhalte präformiert, verdeutlichen auf geradezu atemberaubende Weise heute die digitalen Medien, die neue Wissenshorizonte eröffnen, dabei überkommene Sinnvermutungen dramatisch in Frage stellen, indem sie Umgangsweisen und Wertschätzungen verändern. Ein Zurück zu vertrauten Ufern lassen die neuen Kommunikationsverhältnisse nicht zu. Medien sind danach keine neutralen Werkzeuge, die allein im Sinne ihres Benutzers gebraucht werden, sondern gedankenanregende sowie Kommunikation vorantreibende/verändernde/gestaltende Kraft. Ihre Analyse kann das opake Medium - die >Materialität der Kommunikation « mit ihren Folgen - sichtbar werden lassen. Dies öffnet Sinnsuchenden den Blick über bloße Inhalte hinaus für weitergehende `Botschaften`, die sich reflexiv, konstruktiv wie kreativ mitunter auch gegen die Medialität wenden lassen. 
Musiktheorie hat sich, die bisherigen Ausführungen zusammenfassend,

1. mit dem Klangphänomen,

2. mit dem historischen Kontext,

3. mit den Medialitäten und,

4. um zu vertretbaren Urteilen zu kommen, die sich nachvollziehbar darstellen und doch dabei anerkennen, dass sie auch jederzeit ganz anders hätten ausfallen können und auch werden, mit dem eigenen Reflexionsgrund zu befassen.

Musiktheorie zur Zeit ist von einem Mangel an Zeitgemäßheit ausgezeichnet, indem sie den genannten Erfordernissen theoretischer Betrachtung von Musik nicht hinreichend gerecht wird und überdies ihr Blick weitgehend - aus einer, wie es scheint, selbst verursachten Not heraus - der Vergangenheit mehr verbunden bleibt. Die Not und der Blick zurück gründen darin, dass die Musiktheorie im Verlaufe des 20. Jahrhunderts schlicht ihren `Gegenstand ‘ verloren hat, der sich weniger in dem erklingenden Phänomen, sondern eher in Notenpapier und Notenwelt erfüllte, aus deren Zusammenspiel heraus logische Zusammenhänge, vermeintliche Gesetzmäßigkeiten oder unterstellte »Tendenzen des Materials« (Theodor W. Adorno) abgeleitet werden mochten.

Der Entwicklungs- oder Fortschrittsgestus ist heute ebenso Geschichte wie der damit verbundene Glaube, zunehmende Komplexität und Differenzierung würden wachsende ästhetische Qualität implizieren und eines fernen Tages darin sich erfüllen. Das ist das eine, das andere: Das Prinzip der Präskription, das sich aus der Notenschrift sukzessiv ehedem entwickelte, ist heute weitgehend wieder aufgehoben, wo Musikschaffende ihren individuellen Privatphilosophien folgend - Interpreten große und größte Freiheitsgrade beim Deuten von Partituren einräumen, eigene Zeichenwelten ersinnen, die über spezifische Kompositionen hinaus kaum Verbindlichkeit haben, oder auch gänzlich auf das Aufschreiben von Musik verzichten und die Aufschreibarbeit gleich dem digitalen Speicher überantworten.

Die Notenschrift ist im 21. Jahrhundert nicht mehr das Leitmedium der Musik, das sie für wenige Jahrhunderte war. Die digitalen Speicher haben ihr diesen Rang längst abgelaufen, auch deshalb, weil sie nicht nur genauer und nicht bloß symbolisch aufzuschreiben verstehen, sondern auch das zwischen den Intervallen Erklingende diskret berücksichtigen. Die Notenschrift ist dazu einerseits in ihrer symbolischen Codierung als auch andererseits in ihrer eingeschränkten Distinktion zur Diskretion nicht oder nur unzureichend in der Lage. Der Musiktheorie ist - wo denn der Blick weiterhin prominent auf Notenwerke gerichtet bleibt - diese Entwicklung zum manchmal auch existenziellen Problem geworden. Und die selbstverursachte Not liegt darin begründet, dass der Blick allzu häufig starr gerichtet bleibt auf das ins Glied zurückgetretene Medium Notenschrift.

Eine zeitgemäße Musiktheorie würde

1. der digitalen Aufschreibarbeit mehr Aufmerksamkeit widmen,

2. einer aus dem neuen Leitmedium heraus geborenen Musik angemessenes AnalyseInstrumentarium entwickeln und 
3. die Analyse von Notenwelten, die fraglos für die Betrachtung der Musik der Tradition oder für die Betrachtung von Musik aus ganz speziellem Blickwinkel von Bedeutung ist, nichtsdestoweniger hintenanstellen oder zumindest relativieren.

Musiktheorie hat also nicht weniger als sich selbst neu zu erfinden. Eine solche NeuErfindung oder zumindest Neu-Orientierung würde auch Forschung zum Kernmerkmal von Musiktheorie wieder machen können, wo sie heute ihre Relevanz an musikpädagogischen (und auch an musikwissenschaftlichen) Instituten vornehmlich in der Lehre beweist. In ihrer tradierten Ausrichtung dagegen ist Forschung in der Musiktheorie - auf den Punkt gebracht - eher von leerlaufender selbstgenügsamer Selbstreferentialität bestimmt, die schlicht folgenlos bleibt und bleiben muss.

Bei allen kritischen Untertönen gilt es aber beschließend auch festzuhalten: Die Musiktheorie in ihrer jetzigen Ausprägung ist gleichwohl auch heute für die Lehrerausbildung an musikpädagogischen Instituten nicht nur hilfreich, sondern unverzichtbar, wenn es darum geht, - unter Berücksichtigung des oben genannten erweiterten Blickwinkels sowie unter Ausklammerung der Vermittlung eines zuletzt nicht begründbaren Soseins von Musik - spezifische musikalische Grundlagen in Harmonie- und Formenlehre zu legen, die im Musikunterricht nützlich sind, wenn es um die Auseinandersetzung mit der Musik der Vergangenheit geht. Ihre Grenzen erreicht eine solche Musiktheorie in der Lehre aber schnell dort, wo maßgebliche, die jüngere musikalische Vergangenheit oder die Gegenwartsmusik berührende Schwerpunkte im Musikunterricht zum Thema werden. Mit der Erweiterung ihres Themen- und Forschungsspektrums wäre sie der Musikpädagogik daher ein noch größerer Gewinn.

\section{Literatur}

Geck, Martin (2009), Wenn der Buckelwal in die Oper geht, München: Siedler.

Gumbrecht, Hans Ulrich und Karl Ludwig Pfeiffer (1988), Materialität der Kommunikation, Frankfurt a.M.: Suhrkamp. 